\title{
Estrategia en la supuración de pleura y pulmón según el riesgo quirúrgico
}

\author{
Strategy in pleural and lung suppuration depending on the surgical risk
}

\author{
Orestes Luis Mederos Trujillo,* Orestes Noel Mederos Curbelo,** \\ Juan Carlos Barrera Ortega,*** Juan Antonio Castellanos González,*** \\ Carlos Alberto Romero Díaz,*** Genaro Cruz Caloca****
}

\begin{abstract}
Palabras clave:
Riesgo quirúrgico, supuración de pleura y pulmón, resección, drenaje percutáneo, neumostomía, ventana pleurocutánea.
\end{abstract}

Key words: Surgical risk, pleural and lung suppuration, resection, percutaneous drainage, pneomostomy, pleurocutaneous window.

* Especialista de I grado en Cirugía.

** Especialista de II grado en Cirugía. Doctor en Ciencias, Profesor e Investigador Titular, FACS.

*** Especialista de II grado en Cirugía General. Profesor Auxiliar.

**** Residente de tercer año de Cirugía.

Hospital Universitario Manuel Fajardo Rivero. La Habana, Cuba

\section{RESUMEN}

Introducción: La necesidad de enfrentar problemas torácicos infecciosos complejos con técnicas de alto riesgo en pacientes de edad avanzada y mayor morbilidad asociada aumenta el riesgo quirúrgico. Objetivo: Describir la evolución de los enfermos intervenidos con supuración de pleura y pulmón aplicando una estrategia de selección quirúrgica según el riesgo quirúrgico. Material y métodos: Se realizó un estudio de corte trasversal de 139 pacientes, el $75.5 \%$ con supuración de pleura y el 24.5\% de pulmón, cuyo tratamiento fue quirúrgico. Se definieron pacientes de alto riesgo a los ASA III y IV, con afectación cardiovascular y respiratoria. Resultados: Predominaron los enfermos mayores de 60 años y sexo masculino. En los ASA I y II se realizó con más frecuencia resección en la supuración pulmonar y decorticación en la pleura, en los ASA III y IV y alto riesgo predominó la neomostomía en la supuración pulmonar y el drenaje con lavado y la ventana torácica en la pleura, todas con anestesia local. Los sometidos a intervenciones mayores tuvieron un riesgo de complicarse 2.36 veces mayor, con una correlación negativa débil entre estadía hospitalaria y edad; la mayoría de las complicaciones fueron clínicas, con una baja mortalidad. Conclusión: La implementación de una estrategia quirúrgica basada en el riesgo quirúrgico demostró ser un método eficaz para el tratamiento y evolución de los enfermos con supuración de pleura y pulmón.

\section{ABSTRACT}

Introduction: The need to address complex, infectious thoracic problems with high-risk techniques on elderly patients increases the surgical risk. Objective: To describe the evolution of patients subjected to drainage of lung and pleura applying a strategy of surgical selection according to the surgical risk. Material and methods: A cross-sectional study of 139 patients was conducted, $75.5 \%$ with suppuration of pleura and $24.5 \%$ of lung, whose treatment was surgical. High-risk patients ASA III to IV were determined, with cardiovascular and respiratory disease. Results: Male patients and those 60 years and older dominated. In those ASA I and II, resection of the pulmonary suppuration and decortication of the pleura was performed more frequently; in those ASA III and $I V$, and high-risk, pneomostomy on the lung suppuration and the drainage with washing up and thoracic window in the pleura dominated, all under local anesthesia. Those submitted to major interventions were at 2.36 times greater risk of complication, with a weak negative correlation between hospital stay and the patient's age; most complications were clinical, with low mortality rate. Conclusion: The implementation of a surgical strategy based on surgical risk proved to be an effective method for the treatment and outcome of the patients with lung and pleura suppuration.

\section{INTRODUCCIÓN}

$\mathrm{L}$ as enfermedades infecciosas del tórax acompañan a la humanidad desde épocas remotas. Factores como edad, tabaquismo, función respiratoria deteriorada, insuficiencia cardiaca, padecimientos coronarios, diabetes mellitus, vasculopatía periférica y problemas nutricionales incrementan el riesgo y la morbimortalidad; la necesidad de enfrentar estos pro- blemas en personas de la tercera edad obliga a variar la conducta quirúrgica. ${ }^{1-6}$ La supuración de la pleura y el pulmón representa un $17 \%$ de los enfermos quirúrgicos en Estados Unidos de Norteamérica; en algunas series fallece el 10\% de los operados por absceso; en la supuración pleural en enfermos de riesgo puede llegar a un 23\%. ${ }^{13,5}$ En Cuba, por el envejecimiento poblacional, ha aumentado su frecuencia. El fracaso de los antimicrobianos en ancianos con 
afecciones sistémicas e inmunodeprimidos que no soportan intervenciones como decorticación, pleurectomía o resección ${ }^{7}$ obliga a una estrategia que disminuya el riesgo y permita eliminar la colección y combatir la infección ${ }^{1,2}$ en el absceso; la resección, aunque es el tratamiento ideal, sólo es soportada por el $45 \%,{ }^{1-3}$ lo que ha motivado a retomar la ventana y la pleurostomía con lavado en empiema y el drenaje percutáneo en el absceso. ${ }^{3-6,8}$

\section{MATERIAL Y MÉTODOS}

Se realizó un estudio observacional descriptivo de corte trasversal con el objetivo de registrar la evolución de los enfermos intervenidos por supuración de la pleura y el pulmón en el Hospital Universitario Manuel Fajardo durante 1998-2013 utilizando una estrategia según el riesgo quirúrgico. El universo está integrado por 139 enfermos, 105 con colecciones en pleura y 34 en pulmón. En la evaluación del riesgo analizamos la edad, el problema quirúrgico y el tratamiento, utilizando la escala del estado físico de la Sociedad Americana de Anestesiología (ASA), la clasificación de Karnofsky y el índice de riesgo nutricional, cardiovascular y respiratorio. Los criterios de exclusión fueron el ASA grado V y VI. Se consideró alto riesgo el ASA III y IV, desnutrición grave, afectación cardiovascular y respiratoria. Se definió como "cirugía de menor riesgo" la realizada en poco tiempo, con anestesia local y sin toracotomía. Con el registro de datos se hizo una base de datos en el programa SPSS 18.1, donde fueron analizados. Se utilizó la estadística descriptiva expresando los resultados en valores absolutos y relativos. Para el análisis de las variables nu-

\begin{tabular}{|c|c|c|c|c|}
\hline \multicolumn{5}{|c|}{$\begin{array}{l}\text { Cuadro I. Distribución de pacientes según } \\
\text { enfermedad y ASA (I, II, III, IV). }\end{array}$} \\
\hline Enfermedad & I & II & III & IV \\
\hline Empiema & 30 & 48 & 25 & 2 \\
\hline Absceso de pulmón & 2 & 9 & 2 & 2 \\
\hline Bulla infectada & 0 & 3 & 3 & 1 \\
\hline Tumor abscedado & 0 & 8 & 0 & 0 \\
\hline Bronquiectasia infectada & 1 & 3 & 0 & 0 \\
\hline Total & 33 & 71 & 30 & 5 \\
\hline
\end{tabular}

méricas, se emplearon medidas de tendencia central y de dispersión; las variables categóricas se evaluaron mediante frecuencia absoluta y porcentaje. Se llevó a cabo la prueba de dependencia $\chi^{2}$ para evaluar la asociación entre variables, la prueba de correlación de Pearson para variables numéricas y de Spearman para variables categóricas, siempre para un intervalo de confianza del $95 \%$ y un error del $3 \%$.

\section{RESULTADOS Y DISCUSIÓN}

Predominó el sexo masculino (71.2\%); el 52.5\% tuvo más de 60 años, siendo éstos más propensos a enfermedades neumonías y los derrames que conducen a supuraciones. La supuración en pleura fue de $75.5 \%$ y en pulmón, $24.5 \%$; destaca en este grupo el absceso primario de pulmón, con el $44 \%$, seguido por neoplasia abscedada, con el 23\%. La mayoría fueron clasificados como ASA II (51.07\%), seguido por ASA I (23.7\%) y ASA III y IV (25.1\%) (Cuadro I, Figuras 1 y 2).

A todos los empiemas se les realizó pleurostomía, excepto a los organizados; en 18 enfermos fue el único tratamiento utilizado, el $75 \%$ tenían una o más de estas características: enfermos geriátricos, malnutrición, inmunodeprimidos o con patologías asociadas. Las técnicas más usadas, excluyendo la pleurostomía, fueron la resección, la ventana y la decorticación. De los 27 enfermos con supuración pleural —todos mayores de 60 años y ASA III y IV_- a 20 se les hizo una ventana torácica; la decorticación se llevó a cabo en el 46.5\% de los pacientes ASA I y II.

En la supuración pulmonar, más de la mitad fueron tratados con resecciones típicas; empleamos la lobectomía en el ASA I y II, evitando la resección atípica con sutura pulmonar para disminuir el riesgo de dehiscencia. La técnica de Monaldi o neumonostomía se realizó en ocho enfermos, cinco en ASA III y tres en IV; de ellos, cuatro por absceso de pulmón y cuatro por bullas infectadas (Cuadro II, Figuras 3 y 4).

De existir problemas en el drenaje con la pleurostomía, adicionamos el lavado pleural con solución salina y fibrinolíticos, utilizándolo en 18 enfermos: 11 en ASA I y II, y 7 en ASA III, con resolución en 11. En el alto riesgo, ASA III y IV, en etapas fibrinopurulenta y organizada, 


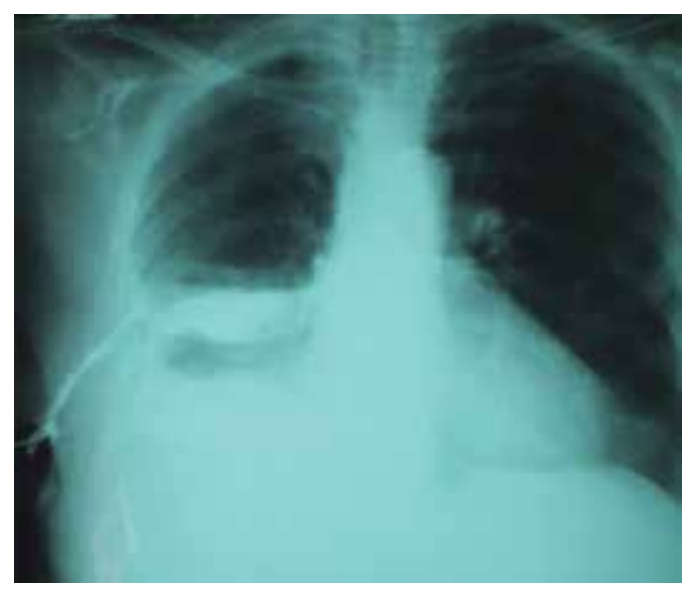

Figura 1. Empiema y fístula. Tratamiento con ventana pleurocutánea.



Figura 2. TAC de absceso de pulmón.

Cuadro II. Distribución según técnica quirúrgica y ASA.

\begin{tabular}{lrrrr} 
Técnica quirúrgica & I & II & III & IV \\
\hline Pleurostomía & 12 & 6 & 0 & 0 \\
Pleurostomía y lavado & 5 & 6 & 7 & 0 \\
Ventana pleurocutánea & 0 & 0 & 18 & 2 \\
Decorticación temprana & 6 & 8 & 0 & 0 \\
Decorticación tardía & 4 & 12 & 0 & 0 \\
Decorticación clásica & 3 & 16 & 0 & 0 \\
Lobectomía & 3 & 20 & 0 & 0 \\
Neumonectomía & 0 & 3 & 0 & 0 \\
Monaldi & 0 & 0 & 5 & 3 \\
Total & 33 & 71 & 30 & 5
\end{tabular}

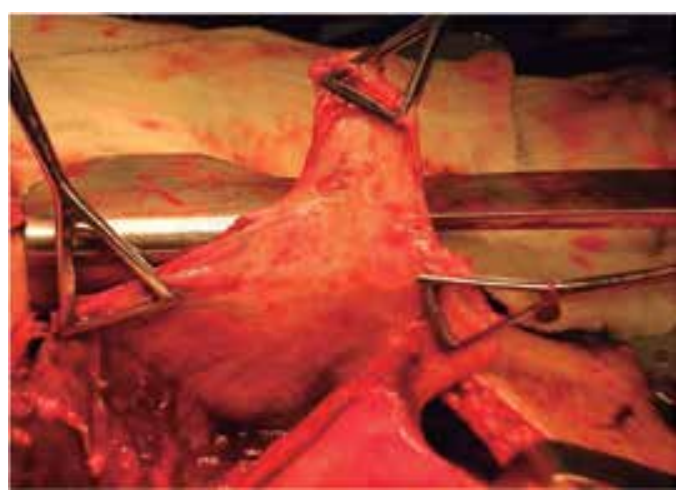

Figura 3. Decorticación clásica.

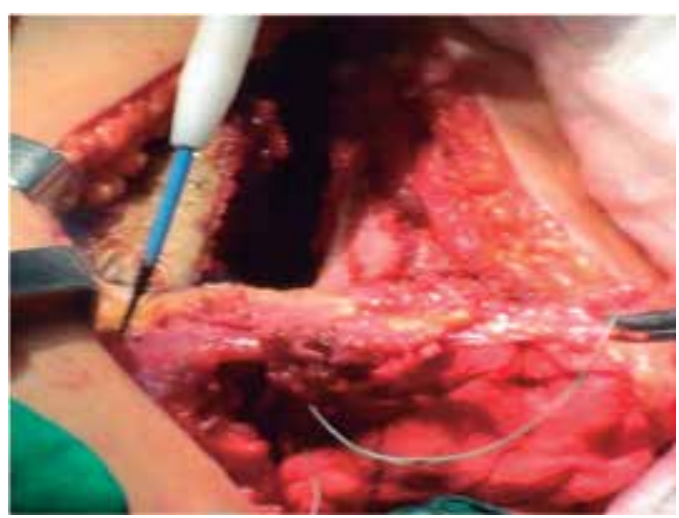

Figura 4. Neumonectomía por absceso con tratamiento del bronquio con colgajo de músculo intercostal.

se utilizó la ventana torácica y la pleurostomía con pleuroclisis, suspendiendo el lavado al verificar tres cultivos negativos y comprobar expansión pulmonar.

La mayor parte de las complicaciones se presentaron en el ASA II (42.4\%); los pacientes de bajo riesgo se complicaron en un $65.6 \%$, mientras que los de alto riesgo se complicaron en un $89.8 \%$. La razón de prevalencia de las complicaciones de los pacientes de alto y bajo riesgo fue de 0.79 . El riesgo de complicarse los pacientes de bajo riesgo fue de 0.27 veces el de los de alto riesgo, con un intervalo de confianza entre 0.091201 y 0.858432 . La complicación más común fue la neumonía nosocomial, 8.3\% (Cuadro III).

La serie incluyó todas las complicaciones locales y generales; la mayoría de las complicaciones se presentaron en las resecciones, destacando la descompensación de enferme- 


\begin{tabular}{|c|c|c|c|c|}
\hline & $\begin{array}{r}\text { ro III. } \\
y\end{array}$ & $\begin{array}{l}\text { ución } \\
\text { le los }\end{array}$ & mplica & \\
\hline \multirow{2}{*}{$\begin{array}{l}\text { Compli- } \\
\text { caciones }\end{array}$} & \multicolumn{2}{|c|}{ Riesgo } & \multirow[b]{2}{*}{ Total } & \multirow[b]{2}{*}{ p } \\
\hline & Bajo & Alto & & \\
\hline Sí & 67 & 33 & 100 & 0.0139 \\
\hline No & 36 & 3 & 39 & \\
\hline Total & 103 & 36 & 139 & \\
\hline
\end{tabular}

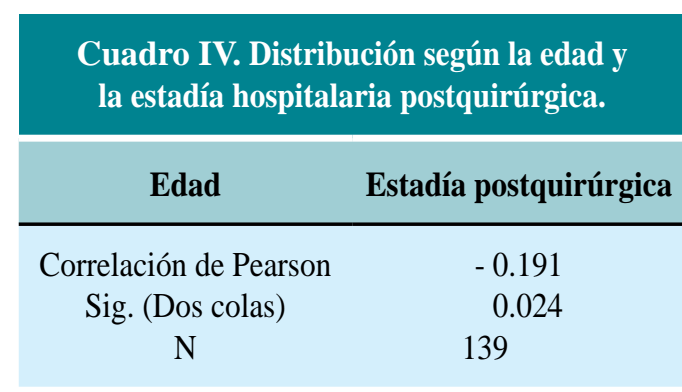

dades asociadas (la mayoría, cardiovascular y respiratoria). Las complicaciones de poca gravedad (seromas, hematomas o infección del sitio quirúrgico) fueron tratadas ambulatoriamente, con poca estadía, y las severas - como empiema o atelectasia- requirieron más estadía. El estudio evidencia esta relación, pues las pruebas estadísticas donde se relacionó la edad con la estadía aplicando la correlación de Pearson y Spearman muestran que existe correlación débil en sentido inverso: a medida que aumenta la edad disminuye la estadía, con un $95 \%$ de certeza (Cuadro IV).

Los enfermos con intervenciones mayores tuvieron un riesgo de complicarse 2.36 veces más alto que los de intervenciones de menor envergadura, con un intervalo de confianza entre 1.113264 y 5.039843 . En la estadística inferencial mediante $\chi^{2}$ encontramos dependencia entre el ASA y las complicaciones, con mayor proporción de complicaciones en ASA I y II en relación con ASA III y IV, resultado de la estrategia de realizar procederes de menor riesgo en los enfermos con alto riesgo, y disminuir el riesgo al utilizar anestesia local. Los pacientes de bajo riesgo presentaron menos probabilidades de complicarse, con un índice de confianza entre 0.091201 y 0.858432 , siendo el riesgo de complicarse en estos pacientes 0.27 veces el de los de alto riesgo; la probabilidad de complicarse según la estadística inferencial fue mayor (casi cuatro veces) en los pacientes de alto riesgo que en los bajo riesgo. No encontramos un estudio que realizara conducta según la clasificación de pacientes en alto y bajo riesgo; en las series revisadas se llevan a cabo procederes menores con anestesia local a pacientes sépticos con alto riesgo, defendiendo la neumonostomía en ancianos de mal estado. En nuestra serie se logró resolución del cuadro séptico en el anciano sin mortalidad, utilizando variantes sencillas y tolerables. La mitad de las neumonectomías presentaron alguna complicación, la mayoría relacionada con enfermedades asociadas; algunos autores plantean que dos de cada cinco de estas resecciones presentan alguna complicación, otros las elevan a un 50\% si incluyen el dolor torácico crónico.

La estadía hospitalaria postoperatoria fue menor en los de alto riesgo, lo que indica que las complicaciones fueron menos graves y la mayoría fueron solucionadas de forma ambulatoria. Tuvimos un fallecido (0.71\%): un enfermo con una neoplasia de pulmón abscedada y un ASA II a quien se le realizó resección pulmonar; murió en terapia al quinto día por un embolismo pulmonar. Los enfermos con empiemas de bajo riesgo en quienes se realizó un proceder de mayor riesgo como decorticación sobrevivieron y se fueron de alta con expansión pulmonar. Más de la mitad de los de alto riesgo tratados con un proceder menos invasivo presentó complicaciones, sin fallecidos en este grupo.

\section{DISCUSIÓN}

El predominio en hombres de las supuraciones de pleura y pulmón está asociado a factores de riesgo como el hábito de fumar y el alcoholismo. El empiema fue la supuración pleural más frecuente debido a que los derrames son el $18 \%$ de las enfermedades respiratorias y en la mayoría se realiza toracocentesis con fines diagnósticos-terapéuticos, siendo la infección posterior a ésta la segunda causa del empiema exudativo. En el absceso de pulmón, el posible éxito del tratamien- 
to ${ }^{9-11}$ antimicrobiano obliga a mantener esta terapéutica por 6-8 semanas.

La realización de una pleurostomía en el empiema es un principio básico en el tratamiento; la mayoría de los autores la defienden como la posible solución en fase aguda. ${ }^{2,12} \mathrm{Se}$ exceptúa de este tratamiento inicial el empiema organizado.

Los procederes con tiempo quirúrgico mayor de tres horas y gran pérdida de sangre aumentan el riesgo quirúrgico al 5\%, y el sitio quirúrgico, la magnitud de la lesión tisular, la inanición, el dolor y la inmovilidad son factores de alto riesgo que aumentan en ancianos; la mayoría de los intervenidos por afecciones torácicas son fumadores y presentan, además, enfermedades respiratorias previas. La toracotomía aumenta el riesgo al producir alteraciones fisiopatológicas por la ventilación de un solo pulmón, y a pesar de un preoperatorio correcto, no podemos suprimir todas las enfermedades asociadas ni la edad, por lo que debemos disminuir el riesgo realizando un proceder quirúrgico menor que pueda soportar el enfermo. ${ }^{1-6,10,11,13-15}$

La decorticación con pleurectomía o abrasión pleural es de elección en la fase fibrinopurulenta y organizada; ${ }^{1,15}$ la realizamos en la mitad de los enfermos, todos en ASA I y II, sin haber tenido fallecidos en la serie. Se informa una mortalidad hasta del $20 \%$ en pacientes que supuestamente la toleraban. Algunos artículos defienden la decorticación por videotoracoscopia o lavados con soluciones proteolíticas en enfermos con mal estado físico y nutricional. ${ }^{12,16-19}$ En la supuración pleural ASA III y IV con cavidades purulentas aisladas por bandas de fibrina y/o pulmón colapsado preferimos la ventana pleural con anestesia local. Todos los de la serie egresaron vivos; en los casos sin cavidad purulenta aislada se realizó pleurostomía.

Aunque hay autores que en el alto riesgo esperan a que mejore el estado general mediante pleurostomía y antimicrobianos para realizar la intervención definitiva, pensamos que en el anciano, por el desgaste biológico, depresión del sistema inmune, disfunción del sistema neurohormonal, trastornos de la nutrición y enfermedades asociadas, el no adoptar una conducta quirúrgica precoz que elimine el foco séptico empeora las condiciones. ${ }^{1-6,19}$
El riesgo quirúrgico presenta tres componentes: el paciente, la enfermedad quirúrgica y la intervención propuesta. Trabajamos en el componente paciente controlando las enfermedades asociadas, mejorando el estado nutricional y general. En la supuración de pulmón se realizaron resecciones en pacientes de bajo riesgo y neumonostomía con sonda de balón (Monaldi) en los de alto riesgo; la diferencia de intervención explica que las complicaciones aparecieran 2.36 veces más en los enfermos con variantes quirúrgicas más complejas; la conducta con procederes de menor riesgo fue obligada (volumen espiratorio forzado en un segundo menor de 0.6 litros); es conocido que sólo en el $60 \%$ de los pacientes con abscesos se puede realizar la resección. ${ }^{1,18}$

Realizamos la decorticación en enfermos con buen estado, y aun así aparecieron complicaciones. Pudimos comprobar una correlación negativa débil entre la estadía hospitalaria y la edad para un 95\% de certeza; al aumentar la edad disminuyó la estadía. ${ }^{19}$

\section{CONCLUSIONES}

Predominaron los enfermos mayores de 60 años y sexo masculino; en los ASA I y II se realizó con más frecuencia resección pulmonar en la supuración pulmonar y decorticación en la pleural; en los ASA III y IV y alto riesgo predominó la neumostomía en la supuración pulmonar y el drenaje con lavado y la ventana torácica en la pleural, todas con anestesia local. La mayoría de las complicaciones fueron clínicas, con una baja mortalidad. La implementación de una estrategia quirúrgica basada en el riesgo quirúrgico demostró ser un método eficaz para el tratamiento y evolución de los enfermos con supuración de pleura y pulmón.

\section{Agradecimientos}

Los autores agradecen la colaboración de los alumnos ayudantes de cirugía Jorge González Lara y Orlando Noel Mederos Trujillo.

\section{REFERENCIAS}

1. Mederos Curbelo ON. Cirugía torácica. Comentarios de casos clínico-quirúrgicos. La Habana: Editorial de Ciencias Médicas; 2012. p. 129-143. ISBN: 978-959212-738-8. 
2. Bekele-Jembere B, Mederos-Curbelo ON, ValdésJiménez J, Barreras-Ortega JC, Romero-Díaz CA, Cantero-Ronquillo A. Manejo escalonado en la supuración pleural. Rev Cubana Cir. 2002; 41: 141146.

3. Mederos-Curbelo ON, Del Campo-Abad R, BarrerasOrtega JC, Romero-Díaz C, Cantero-Ronquillo A, Hung-Chang C. Ventana torácica con anestesia local. Alternativa en el enfermo grave con empiema pleural. Rev Arch Cir Gen Dig [Internet]. 2005. (c) Cirugest. [Consultada el 15 de mayo de 2012]. Disponible en: http://www.cirugest.com/revista/2005/06/2005-03-21. $\mathrm{htm}$

4. Mederos-Curbelo ON, Olewasegun-Abiodun A, Mederos-Trujillo OL, Barrera-Ortega JC, Castellanos-González JA. Ventana torácica y anestesia local en la supuración pleural. Rev Cubana Cir [Internet]. 2016; 55. Disponible en: http://scielo. sld.cu/scielo.php?script $=$ sci_arttext\&pid $=$ SO03474932016000100004\&lng $=$ es.

5. Mederos-Curbelo ON, Barrera-Ortega JC, CastellanosGonzález JA, Romero-Díaz CA, Mederos-Trujillo OL. Neumostomía con sonda de balón en colecciones purulentas del pulmón. Rev Cubana Cir. 2014; 53: 145-155.

6. Mederos-Curbelo ON, Barrera-Ortega JC, CanteroRonquillo A, Da Costa JM, Oliva-Anaya VC. La decorticación pulmonar en el empiema pleural. Rev Cubana Cir [Internet]. 2008; 47. Disponible en: http://scielo.sld.cu/scielo.php?script $=$ sci arttext\&pid=S0034-74932008000300002\&lng=es.

7. Aurek W, Makarewicz W, Bobowicz M, Sawicka W, Rzyman W. The treatment of chronic pleural empyema with laparoscopic omentoplasty. Initial report. Wideochir Inne Tech Maloinwazyjne. 2014; 9: 548-553.

8. Fuentes-Valdés E. Técnica de Monaldi para el tratamiento de abscesos pulmonares. Rev Cubana Cir [Internet]. 2010; 49. Disponible en: http://scielo. sld.cu/scielo.php?script =sci_arttext $\&$ pid $=$ S003474932010000200008\&lng=es.

9. Bartlett JG. How important are anaerobic bacteria in aspiration pneumonia: when should they be treated and what is optimal therapy. Infect Dis Clin North Am. 2013; 27: 149-155.

10. Castillo-Aguilera MÁ, Mederos-Trujillo OL, MederosCurbelo ON. Descompensación diabética y lesión torácica ¿Absceso del pulmón? Revista de Portales Médicos.com [Internet]. 2011.

11. Witzke $\mathrm{H}$, Anikin V. Other conditions of the lung (abscesses, inhaled foreign bodies, bullous lung disease, hydatid). Surgery. 2014; 32: 261-265.

12. Nie W, Liu Y, Ye J, Shi L, Shao F, Ying K, et al. Efficacy of intrapleural instillation of fibrinolytics for treating pleural empyema and parapneumonic effusion: a meta-analysis of randomized control trials. Clin Respir J. 2014; 8: 281-291.

13. Guerra M, Neves PC, Martins D, Miranda J, Leal F, Vouga L. Surgery for thoracic empyema: personal experience and current highlights. Rev Port Cir Cardiotorac Vasc. 2012; 19: 21-26.

14. Socci L, Marta HS, López MF. Treatment of postpneumonectomy empyema: goodbye to Clagett's thoracostomy. Cir Esp. 2011; 89: 329-332.

15. Shin JA, Chang YS, Kim TH, Haam SJ, Kim HJ, Ahn CM, et al. Surgical decortication as the first-line treatment for pleural empyema. J Thorac Cardiovasc Surg. 2013; 145: 933-939.

16. Shipulin PP, Koziar ON, BaÄ $\neg$ dan VV, Martyniuk $V A$, Severgin VE, Kiriliuk AA, et al. Application of early videothoracoscopic pulmonary decortication in the treatment of an acute pleural empyema and suppurated clotted hemothorax. Klin Khir. 2010; (10): 29-31.

17. Arango-Tomás E, Cerezo-Madueño F, Algar-Algar FJ, Salvatierra-Velázquez A. Is lung surgery a good option for octogenarians? Cir Esp. 2014; 92: 615-618.

18. Rodríguez $M$, Gómez $M T$, Jiménez MF, Aranda JL, Novoa N, Varela G. The risk of death due to cardiorespiratory causes increases with time after right pneumonectomy: a propensity score-matched analysis. Eur J Cardiothorac Surg. 2013; 44: 93-97.

19. Søgaard M, Nielsen RB, Nørgaard M, Kornum JB, Schønheyder HC, Thomsen RW. Incidence, length of stay, and prognosis of hospitalized patients with pleural empyema: a 15-year Danish nationwide cohort study. Chest. 2014; 145: 189-192.

Correspondencia:

Dr. Cs. Orestes Noel Mederos Curbelo

E-mail: noemed@infomed.sld.cu 